\title{
SELECCIÓN DE WAVELETS MADRE PARA LA CARACTERIZACIÓN DE 5 TIPOS DE LATIDOS CARDIACOS EMPLEANDO LA TRANSFORMADA WAVELET DISCRETA
}

\author{
SELECTION OF MOTHER WAVELET TO CHARACTERIZE 5 KINDS OF HEARTBEATS USING \\ THE DISCRETE WAVELET TRANSFORM.
}

Alejandro José Orozco Naranjoํㅜ, Pablo Andrés Muñoz Gutiérrez ${ }^{1}$

' Universidad del Quindío, Grupo de automatización y máquinas de aprendizaje (GAMA). alejorozco@ingenieros.com,pamunoz@uniquindio.edu.co.

Recibido: Marzo 5 de 2012

Aceptado: Junio 1 de 2012

*Correspondencia del autor. Facultad de ciencias básicas, Programa de tecnología en electrónica, Universidad del Quindío, Armenia

(Quindío); correo electrónico: pamunoz@uniquindio.edu.co

\section{RESUMEN}

Se documenta la evaluación de metodologías para la selección de aquellas wavelets madre que permitan caracterizar señales cardiacas de mejor manera, a partir del análisis de los coeficientes de aproximaciones y detalles, obtenidos al aplicar la transformada wavelet discreta a 5 tipos de latidos cardiacos. El indicador que permite la identificación de las wavelets madre, está basado en el análisis de la energía concentrada en las aproximaciones y detalles de los primeros cuatro niveles de descomposición. Los vectores de características generados se ingresaron en Máquinas de Soporte Vectorial con Kernel Polinomial y Función de base radial, permitiendo cuantificar la capacidad de caracterización con las wavelets madre que lograron indicadores energéticos de interés, a partir de los resultados de clasificación. La menor cantidad de errores fueron obtenidos utilizando la wavelet madre Biortogonal Inversa de orden 3.1 (rbio3.1), la cual no es mencionada comúnmente en divulgaciones científicas, con una máquina de soporte vectorial de Kernel Polinomial, cuyo error porcentual fue de $2,71 \%$.

Palabras clave: Clasificación, Identificación de patrones, Indicadores energéticos, Máquina de soporte vectorial, Transformada wavelet discreta, Wavelet madre.

\begin{abstract}
:
It's documented the review of a methodology to select those mother wavelet that allow to characterize heartbeats by a best way, from the analysis of the coefficients of approximations and details, obtained by applying the discrete wavelet transform to 5 kinds of heartbeat. The indicator which allows the mother wavelets identification is based on analysis of concentrated energy in approximations and details, of the first four levels of decomposition. The generated feature vectors were entered into Support Vector Machine with Polynomial kernel and radial basis function, allowing quantifying the characterization capability of mother wavelets that achieved interesting energy indicators, from the results of classification. Fewer errors were obtained using the Reverse Biorthogonal mother wavelet of order 3.1 (rbio3.1), which is not considered commonly in research papers, with a support vector machine with polynomial kernel, whose percentage error was $2.71 \%$.
\end{abstract}

Keywords: Classification, Identification of patterns, Energy indicators, Support vector machine, Discrete wavelet transform, Mother wavelet. 


\section{INTRODUCCIÓN}

Con la incorporación de herramientas de procesamiento e identificación de señales al campo médico, se ha facilitado el diagnóstico en un sinnúmero de especialidades, entre ellas se encuentra la cardiología, disciplina que se ocupa del comportamiento cardiaco y sus efectos en todo el organismo, por lo que es necesario el desarrollo de metodologías y técnicas que hagan menos engorroso el análisis de los registros electro-cardiográficos (ECG). Las características presentes en un ECG para la evaluación médica son: Frecuencia y ritmo cardíaco, ejes, complejo QRS y otros. El registro ECG representa en tiempo y voltaje los periodos de despolarización y re-polarización del miocardio $(1,2)$.

En los últimos años se han adelantado investigaciones por parte de diversos grupos de investigación, que se ocupan de la identificación de anomalías cardiacas, proponiendo la utilización de técnicas de procesamiento de señales, caracterización y clasificación de patrones (3-5) y otras se han encargado de hacerle frente a problemas muy específicos, por ejemplo la eliminación de ruido propio de las señales Electrocardiográficas (ECG) (6). Aunque existen varias metodologías para el análisis de señales electrocardiográficas, la transformada wavelet ha proporcionado un alto grado de confiabilidad, articulándose con máquinas de aprendizaje para detectar un evento cardiaco anormal. La transformada wavelet hace posible la descomposición de una señal en un conjunto diferente de señales restringidas para ciertas bandas de frecuencia (7). La transformada wavelet discreta ha permitido analizar gran variedad de señales de naturaleza biológica en tiempo y frecuencia, en particular permitiendo visualizar componentes cardiacos no tradicionales de los latidos, aportándose características para ser utilizadas como información en los sistemas de aprendizaje (8). La investigación aquí propuesta se enmarca especialmente dentro de una fase fundamental previa a la clasificación de patrones, conocida como caracterización, a través de la cual se obtienen características propias de ejemplos de determinadas clases, para que estos sean utilizados en el aprendizaje, por sistemas con cierto grado de inteligencia. Investigaciones previas se han centrado en utilizar wavelets madre como son symlet y daubechies para la caracterización de la información, con las cuales se han obtenido resultados satisfactorios de clasificación $(3,5,9)$ pero pocas veces se clarifica si realmente se evaluaron otras wavelet madre o simplemente se utilizaron estas solo porque son las más referenciadas en la literatura, aún cuando se cuenta con una cantidad considerable de wavelets madre, que pueden ser evaluadas procurando una mejor caracterización que permita la clasificación de patrones y en este caso, de latidos cardiacos mucho más eficaz. Entre los trabajos de investigación que se puede identificar un estudio serio de la evaluación de wavelets madre, según el tipo de señal a utilizar se pueden destacar $(10,11)$ en los que a partir del análisis energético de los coeficientes de aproximaciones y detalles obtenidos de cada nivel de descomposición, identifican las wavelet madre que mejor representan las señales analizadas, ambos trabajos se centran en la identificación de fallos en máquinas rotatorias. Los errores de clasificación típicamente encontrados al emplear máquinas de soporte vectorial con una fase previa de caracterización basada en la transformada wavelet discreta (DWT) se encuentran entre el $3 \%$ y $5 \%(5,9)$.

El presente artículo se encuentra estructurado en cuatro secciones que describen la metodología aplicada, los resultados obtenidos al analizar su distribución energética y validar las caracterizaciones realizadas con Máquinas de Soporte Vectorial y finalmente los apartados de conclusiones y bibliografía.

\section{MATERIALES Y MÉTODOS}

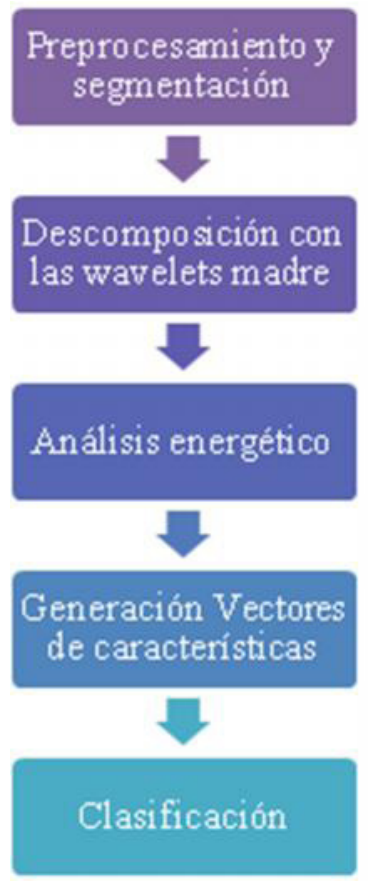

Figura 1. Metodología para evaluación de técnica de clasificación a partir del cálculo de energía de los coeficientes wavelet. Fuente propia.

En el diagrama bloques de la Figura 1 se describe la metodología seguida para el reconocimiento de las señales 
cardiacas, a partir del análisis energético de los coeficientes obtenidos con la transformada wavelet discreta.

\section{A. Pre-procesamiento y segmentación}

En este ítem se incluye todo el tratamiento previo a la extracción de características con la DWT realizado a los latidos cardiacos. Los latidos cardiacos utilizados provenían de la base de datos MIT BIH Arrhythmya Database disponible en Physionet, allí se cuenta con registros electrocardiográficos de larga duración que están debidamente etiquetados, facilitando un posterior análisis a partir de herramientas software, entiéndase por etiquetados a la referencia temporal y al uso de indicadores numéricos (Anotaciones) que permitían conocer el tipo del latido cardiaco dentro del registro completo (12). Aunque había dos derivaciones disponibles en la base de datos, se optó por emplear la V1 debido a su amplio uso en el diagnostico médico. Después de un análisis de la cantidad de latidos cardiacos por cada clase en la base de datos, se seleccionaron los siguientes tipos de latidos: Normal, Bloqueo de rama izquierda (LBBB), Bloqueo de rama derecha (RBBB), Contracción ventricular prematura( $\mathrm{PVC}$ ) y contracción auricular prematura (APC), de cada clase se tomaron 1180 ejemplos elegidos al azar.

Inicialmente se cargaron los registros ECG completos para que les fueran eliminados componentes interferentes como es el ruido de línea base y el gausiano, lo cual se realizó con filtrado basado en la transformada wavelet discreta (Denoising) (13). Otra etapa de filtrado aplicada fue la eliminación de la línea base, que se hizo con un filtro de media móvil (6). Para la segmentación se dispuso una ventana móvil que permitiera acotar cada pulso cardíaco de interés. La determinación de la ventana se hizo a partir de las anotaciones mencionadas, las cuales permiten identificar en el tiempo la presencia de un latido cardiaco, se estableció un rango intermedio entre el latido actual y el siguiente, así como entre el actual y el anterior, para finalmente delimitar en el tiempo cada latido que fuera de la clase de interés. Las dimensiones de cada latido generado en archivos independientes era de 256 muestras y contenían tanto el comportamiento eléctrico como una referencia temporal, con esto se garantizaría uniformidad en la cantidad de coeficientes generados con cada wavelet madre.

\section{B. Descomposición con las wavelets madre}

En general la Transformada Wavelet, que hace uso de las wavelet madre, es una herramienta matemática que delimita los datos, en diferentes componentes de frecuencia y estudia cada componente a una resolución ubica- da a esa escala. La justificación del uso de Wavelets se basa en el hecho de que la Transformada de Fourier no tiene una buena resolución Tiempo - Frecuencia, pues al analizar la señal lo trata como un todo y finalmente muestra las frecuencias presentes a lo largo de toda la señal, pero no indica el tiempo en que estas ocurrieron. Siendo la transformada wavelet más que apropiada para el estudio de señales no estacionarias como es el caso de las electrocardiográficas. Con la transformada wavelet discreta (DWT) los parámetros de dilatación y traslación $a$ y $b$, ambos toman solamente valores discretos, para $a$ se escoge $a=a_{m}{ }^{0} \operatorname{con} a_{0}>1$, diferentes valores de $m$ corresponden wavelets con diferentes anchos, entonces es de esperarse que la discretización del parámetro de traslación $b$ dependa de $m$, en las frecuencias altas las wavelets son trasladados en pasos pequeños y en frecuencias bajas las wavelets son trasladados en pasos grandes, a fin de cubrir todo el rango del tiempo de la señal, si el ancho del wavelet es proporcional a $a_{\mathrm{m}}^{0}$, entonces la discretización de $b=n b_{0} a_{m}{ }^{0}$, donde $b_{0}$ es fijo y $n \in Z$, luego las wavelets quedarán discretizadas de la forma [1] [2].

$$
\begin{aligned}
& \psi^{m, n}(x)=a_{0}^{-\frac{m}{2}} \psi\left(a_{0}^{-m}\left(x-n b_{0} a_{0}^{m}\right)\right) \\
& \psi^{m, n}(x)=a_{0}^{-\frac{m}{2}} \psi\left(a_{0}^{-m} x-n b_{0}\right)
\end{aligned}
$$

En particular se escoge [3]

$$
\begin{aligned}
a_{0}=2 \text { y } b_{0} & =1 . \text { Así, } \psi^{m, n}(x) \\
& =a_{0}^{-\frac{m}{2}} \psi\left(2^{-m} x-n\right)
\end{aligned}
$$

En la Figura 2 se puede ver la relación de a contra $b$. Esta familia de funciones es llamada el set de expansión wavelet (14). La wavelet madre $\psi$, trae siempre asociada consigo una función escala $\phi$. Con estas dos funciones se puede aproximar cualquier función o señal $f \in L 2$, mediante una de las funciones o mediante ambas, de la forma mostrada en [4]:

$$
f(t)=\sum_{m} \sum_{n} c_{m, n} \phi(t)+\sum_{m} \sum_{n} d_{m, n}(t) \psi
$$




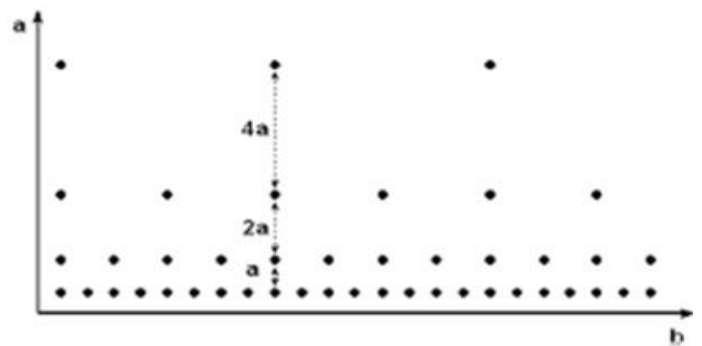

Figura 2. Localización de las wavelets discretas en el espacio tiempo-escala en una malla diádica.Tomada de (12).

Después de contar con los latidos segmentados y dispuestos en archivos independientes se procedió a aplicar descomposiciones con la transformada wavelet discreta a todos los 5900 latidos disponibles (1180 de cada clase) con las wavelet madre mostradas en la Tabla 1. Estas wavelet madre son las disponibles en el software Matlab 2011. Cada wavelet madre se evaluó con diversos órdenes, entiéndase por orden al número que acompaña el prefijo de la wavelet madre, por ejemplo en $\mathrm{db} 2$, la wavelet madre es daubechies y el orden es 2. Cuando el orden sea mayor, habrá una mayor cantidad de cruces por cero en dicha señal de referencia que será relacionada con los latidos cardiacos. Según la teoría wavelet se obtendrán coeficientes de aproximaciones y detalles con mayores amplitudes, cuando haya una mayor similitud entre la wavelet madre y la señal analizada con la DWT. La cantidad de niveles de descomposición aplicados fueron 4. Aunque realizar un análisis directo sobre las amplitudes de los coeficientes de aproximaciones y detalles obtenidos es válido, solo un análisis energético de dichos coeficientes nos permite conocer que tanta información relevante respecto de la señal original, se encuentra contenida en los coeficientes seleccionados, de manera similar a como ocurre con la selección del mejor árbol en la transformada por paquetes wavelet.

\section{Análisis energético}

Teniendo los coeficientes de aproximaciones y detalles obtenidos tras realizar las descomposiciones con las wavelets madre mostradas en la Tabla 1, se procedió a normalizar estos coeficientes para que los valores se encontraran dentro de rangos no muy alejados y se evitara que por picos muy marcados en la información, se incrementara el valor de la energía exageradamente en algunos casos. A la información obtenida se le calculó la energía con un parámetro logarítmico para hacer más notables las variaciones en la información, el cálculo de la energía se hizo con [5]

$$
E\left(s_{i}\right)=\sum_{i} \log \left(s_{i}^{2}\right) \text { con } \log (0)=0
$$

El cálculo se hizo para aproximaciones y para detalles por separado, después se promedió el valor de energías de los 1180 ejemplos de cada clase, es decir, por cada tipo de latido cardiaco se obtuvieron dos promedios, uno de los coeficientes de detalles y el otro de los coeficientes de aproximaciones. Finalmente se promediaron los promedios de energía de las 5 clases por cada wavelet madre utilizada, precisamente porque se buscaba aquella wavelet madre que mejor representara los 5 tipos de

Tabla 1. Wavelets madre utilizadas.

\begin{tabular}{cccccc}
\hline \multicolumn{6}{c}{ Wavelets Madre } \\
\hline bior1.1 & bior3.5 & coif4 & $\mathrm{db} 7$ & rbio2.6 & rbio6.8 \\
bior1.3 & bior3.7 & coif5 & $\mathrm{db} 8$ & rbio2.8 & sym1 \\
bior1.5 & bior3.9 & $\mathrm{db} 1$ & $\mathrm{db} 9$ & rbio3.1 & sym2 \\
bior2.2 & bior4.4 & $\mathrm{db} 10$ & $\mathrm{dmey}$ & rbio3.3 & sym3 \\
bior2.4 & bior5.5 & $\mathrm{db} 2$ & rbio1.1 & rbio3.5 & sym4 \\
bior2.6 & bior6.8 & $\mathrm{db} 3$ & rbio1.3 & rbio3.7 & sym5 \\
bior2.8 & coif1 & $\mathrm{db} 4$ & rbio1.5 & rbio3.9 & sym6 \\
bior3.1 & coif2 & $\mathrm{db5}$ & rbio2.2 & rbio4.4 & sym7 \\
bior3.3 & coif3 & $\mathrm{db6}$ & rbio2.4 & rbio5.5 & sym8 \\
\hline
\end{tabular}

db: Daubechies; bior:Biortogonal; coif: Coiflet;rbio:Biortogonal Inversa; dmey: Aproximación discreta de meyer; sym: Symlet. Fuente propia. 
latidos cardiacos estudiados. Estos promediados de promedios serán más claros al presentarse los resultados.

\section{Generación de vectores de características}

Después de conocer aquellas wavelet madre que mejor representaban a los 5 tipos de señales cardiacas se aplicaron dos esquemas de extracción de características, como los mostrados en la Tabla 2, que en adelante serán referenciados como Esquema 1 y Esquema 2; estos fueron establecidos gracias a experiencias previas con la transformada wavelet discreta, que permitieron identificar estructuras de selección de características que permitían acceder a información relevante para las maquinas de aprendizaje para la posterior etapa de clasificación.
Desempeñándose bien sobre ejemplos no vistos. Para el caso de espacio de entrada dimensional como se muestra en la Figura 3, hay muchos posibles clasificadores lineales que pueden separar los datos, pero hay solo uno que maximiza el margen. Este clasificador lineal es llamado hiperplano de separación óptima.

El hiperplano $(w x)+b=0$ satisface las condiciones [10] $y[11]$

$$
\begin{array}{ll}
\left(w * x_{i}\right)+b>0 ; & \text { Si } y_{i}=1 \\
\left(w * x_{i}\right)+b<0 ; & \text { Si } y_{1}=-1
\end{array}
$$

Tabla 2. Esquemas de extracción de características utilizados.

\section{Esquemas de Extracción de características}

\begin{tabular}{lr}
\hline Esquema 1 & Todos los coeficientes de aproximaciones de niveles 1, 2, 3 y 4 \\
Esquema 2 & Todos los coeficientes de aproximaciones y detalles de niveles 2 y 3
\end{tabular}

\section{E. Clasificación}

Para la fase de clasificación, con la cual se valida cuantitativamente la capacidad de caracterización de las wavelet madre identificadas como las que mejor desempeño muestran, se utilizaron máquinas de soporte vectorial debido sus notables capacidades en el contexto de identificación de patrones y en particular de latidos cardiacos, temática en la cual los autores han venido trabajando desde hace algún tiempo.

Las máquinas de soporte vectorial son un conjunto de algoritmos desarrollados por Vladimir Vapnik, que pertenecen a la familia de clasificadores lineales e introducen funciones núcleo o kernel (15). El problema que se considera, busca separar un conjunto de patrones $\left(x_{1}, y_{1}\right), \ldots,\left(x_{i}, y_{i}\right), \in \mathrm{R}^{n}$ que pertenecen a dos clases etiquetadas como (clase $\mathrm{A} \rightarrow y_{i}=1$; clase $B \rightarrow y_{i}=-1$ ), a través de un hiperplano dado por [6] [7] y [8]:

$$
\begin{gathered}
\{w * x\}+b=0 \\
w \in R^{n} \\
b \in R
\end{gathered}
$$

Donde $\mathrm{W}$ y b son parámetros que se inducen a partir de los ejemplos disponibles correspondientes a la función de decisión [9]

$$
f(x)=\operatorname{sign}(w x+b)
$$

Combinando las dos expresiones en la ecuación y escalando $w$ y $b$ con un factor apropiado, una superficie de decisión equivalente se puede formular como aquella que satisfaga la restricción [12]

$$
\left[\left(w * x_{i}\right)+b\right] \geq 1 \quad i=1,2 . . l
$$

Se puede demostrar que el hiperplano que separa óptimamente los datos en dos clases es que aquel que minimiza el funcional (13)

$$
\Phi(w)=\frac{\|w\|^{2}}{2}
$$

Por lo tanto, el problema de optimización puede ser reformulado como un problema de optimización restringida, usando multiplicadores de Lagrange y su función estaría dada por la identificación de los puntos de silla del funcional de Lagrange (16) dados por [14] [15] [16] y [17].

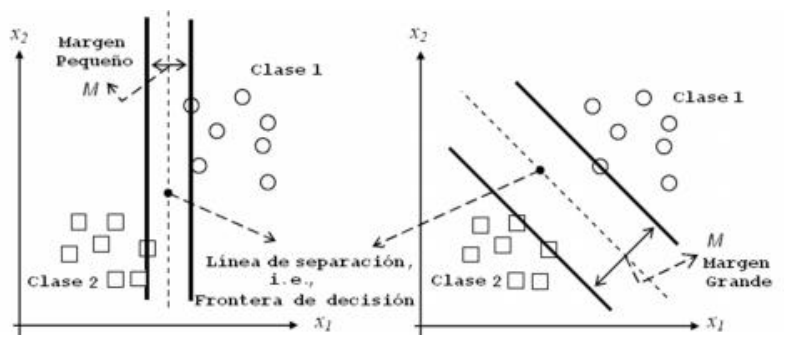

Figura 3. Hiperplano de separación óptima para el caso bidimensional. Tomada de (13). 


$$
\begin{gathered}
L(w, b, \propto)=\frac{\|w\|^{2}}{2}-\sum_{i=1}^{l} \alpha_{i} y_{i}=0 \\
\frac{\partial L}{\partial w}=0 \\
w-\sum_{i=0}^{l} y_{i} \propto_{i} x_{i}=0 \\
w=\sum_{i=0}^{l} y_{i} \propto_{i} x_{i}
\end{gathered}
$$

Se evaluaron máquinas de soporte vectorial (MSV) con kernel polinomial y con kernel función de base radial. La configuración para la MSV con kernel polinomial fue Coeff:1 y Degree: 3.; y para Para la MSV con kernel función de base radial fue C:1 y Gamma:50. Al contar con 1180 ejemplos de cada tipo de latidos, se tomaron aleatoriamente 900 de cada clase de manera aleatoria para la fase de entrenamiento y los restantes 280 para la fase de prueba.

\section{RESULTADOS Y DISCUSIÓN.}

Según la metodología para la identificación de la wavelet madre que mejor caracteriza los latidos cardiacos de los 5 tipos, el primer paso consistía en conocer el valor promedio de la energía contenida en los 1180 ejemplos de cada tipo de latido cardiaco con las todas las wavelet madre. En la Tabla 3 se observan los valores obtenidos con las 5 wavelet madre que mejores resultados mostraron, por mejores se entiende que son los valores de energía más altos.

La wavelet madre que muestra mayores valores de energía con los latidos RBBB es bior 3.3 con las aproximaciones y rbio 3.1 con los detalles; con los latidos PVC es bior 3.3 para las aproximaciones y rbio 3.1 para los detalles; con los Normales es bior 3.7 para las aproximaciones y rbio 3.1 para los detalles; con los LBBB es bior 3.9 para las aproximaciones y rbio 3.1 para los detalles y con los APC es bior 3.3 para las aproximaciones y rbio3.1 para los detalles. Las energías más altas se obtuvieron con la wavelet madre bior 3.7 para aproximaciones con latidos Normales y con la wavelet madre rbio 3.1 para los detalles con latidos Normales. Posteriormente se realizó un promedio de las energías por cada wavelet madre, buscando obtener un valor energético promedio de los 5 tipos de latidos cardiacos, tanto para detalles como para aproximaciones. Los resultados obtenidos se muestran en la Tabla 4.
Se puede apreciar que el mejor promedio de energía con coeficientes de aproximaciones se dio para la wavelet madre bior 3.3 y para los detalles la wavelet madre rbio 3.1. Después con estas 5 wavelets madre que mostraron los resultados más altos de energía, se procedió a realizar la extracción de características utilizando los Esquemas 1 y 2, aplicando 4 niveles de descomposición con cada wavelet madre, y a lo obtenido se le aplicaron los 2 esquemas de extracción de características. Al ingresar los vectores de características a las máquinas de soporte vectorial, ya sea con kernel función de base radial (RBF) o polinomial (Poly) se obtuvieron los resultados mostrados en la Tabla 5 y la Tabla 6, allí se muestran los 3 mejores casos para cada máquina de aprendizaje. Con la máquina de soporte vectorial con kernel polinomial, el menor error de clasificación es de 2,71\%; cifra muy baja si se tiene en cuenta el contexto de clasificación de latidos cardiacos en que se enmarca. Este resultado se logró con la wavelet madre Biortogonal Inversa 3.1 (rbio31), utilizando el esquema de extracción de características 2. Las otras dos wavelet madre que mostraron buenos resultados con esta máquina de aprendizaje, fueron las que tenían información proveniente de la descomposición con la DWT de las wavelets madre Biortogonal 3.9 y Biortogonal 3.7, con errores de clasificación entre $3,42 \%$ y $3,57 \%$ con los esquemas 1 y 2 respectivamente. Con la máquina de soporte vectorial con kernel Función de base radial, el menor error de clasificación es de 3,00\%; que es una cifra baja pero no mejor al caso con la otra máquina de soporte vectorial. Este resultado también se logró con la wavelet madre Biortogonal Inversa 3.1 (rbio31), utilizando el esquema de extracción de características 2; lo que indica que realizó una caracterización apropiada de la información. Las otras dos wavelet madre que mostraron buenos resultados con esta máquina de aprendizaje, fueron las que tenían información proveniente de la descomposición con la DWT de las wavelets madre Biortogonal 2.2 y Biortogonal 3.3, con errores de clasificación entre $3,71 \%$ y $3,85 \%$, ambos con el Esquema 2. 
Tabla 3. Wavelets que mostraron mejores resultados de energía para los 5 tipos de latidos cardiacos

\begin{tabular}{|c|c|c|c|}
\hline WM & Latido & Prom. E. Aps. & Prom. E. Det \\
\hline bior2.2 & RBBB & $2.80 \mathrm{E}-07$ & $2.21 \mathrm{E}-08$ \\
\hline bior2.2 & PVC & 6.77E-07 & $1.35 \mathrm{E}-07$ \\
\hline bior2.2 & NORMAL & $3.24 \mathrm{E}-06$ & 4.65E-07 \\
\hline bior2.2 & LBBB & 4.81E-08 & 6.92E-09 \\
\hline bior2.2 & APC & 1.13E-06 & 8.11E-08 \\
\hline bior3.3 & RBBB & 4.27E-07 & $3.45 \mathrm{E}-08$ \\
\hline bior3.3 & PVC & 8.62E-07 & $3.42 \mathrm{E}-07$ \\
\hline bior3.3 & NORMAL & 4.91E-06 & 7.74E-07 \\
\hline bior3.3 & LBBB & 7.80E-08 & 1.87E-09 \\
\hline bior3.3 & APC & 1.87E-06 & 2.70E-07 \\
\hline bior3.7 & RBBB & 4.03E-07 & $2.59 \mathrm{E}-08$ \\
\hline bior3.7 & PVC & 7.80E-07 & $1.98 \mathrm{E}-07$ \\
\hline bior3.7 & NORMAL & 5.06E-06 & $1.12 \mathrm{E}-06$ \\
\hline bior3.7 & LBBB & 8.99E-08 & 3.52E-09 \\
\hline bior3.7 & APC & 1.71E-06 & $1.56 \mathrm{E}-07$ \\
\hline bior3.9 & RBBB & 3.97E-07 & 3.07E-08 \\
\hline bior3.9 & PVC & 8.10E-07 & $1.64 \mathrm{E}-07$ \\
\hline bior3.9 & NORMAL & 4.73E-06 & $1.46 \mathrm{E}-06$ \\
\hline bior3.9 & LBBB & 9.07E-08 & 5.66E-09 \\
\hline bior3.9 & APC & $1.74 \mathrm{E}-06$ & $1.39 \mathrm{E}-07$ \\
\hline rbio3.1 & RBBB & 2.01E-07 & 9.71E-08 \\
\hline rbio3.1 & PVC & 4.35E-07 & 3.87E-07 \\
\hline rbio3.1 & NORMAL & $1.22 \mathrm{E}-06$ & $1.57 \mathrm{E}-06$ \\
\hline rbio3.1 & LBBB & 3.63E-08 & 3.67E-08 \\
\hline rbio3.1 & APC & 7.35E-07 & 5.29E-07 \\
\hline
\end{tabular}

WM: Wavelet madre. Prom. E. Aps.: Promedio de energía de coeficientes de aproximaciones. Prom. E. Det.: Promedio de energía de coeficientes de detalles. Fuente propia. 
Tabla 4. Promediado de energías provenientes de los 5 tipos de latidos con una sola wavelet madre

\begin{tabular}{ccc}
\hline WM & Prom. E. Aps. 5 latidos & Prom. E. Det. 5 latidos \\
\hline bior2.2 & $1.08 \mathrm{E}-06$ & $1.42 \mathrm{E}-07$ \\
bior3.3 & $1.63 \mathrm{E}-06$ & $2.85 \mathrm{E}-07$ \\
bior3.7 & $1.61 \mathrm{E}-06$ & $3.02 \mathrm{E}-07$ \\
bior3.9 & $1.56 \mathrm{E}-06$ & $3.60 \mathrm{E}-07$ \\
rbio3.1 & $5.27 \mathrm{E}-07$ & $5.25 \mathrm{E}-07$ \\
\hline
\end{tabular}

WM: Wavelet madre. Prom. E. Aps. 5 latidos: Promedio de energía de coeficientes de aproximaciones de los 5 latidos. Prom. E. Det. 5 latidos: Promedio de energía de coeficientes de detalles de los 5 latidos. Fuente propia.

Tabla 5. Resultados de clasificación con la máquinas de soporte vectorial con kernel polinomial

\begin{tabular}{ccc}
\hline \multicolumn{3}{c}{ Resultados de clasificación MSV kernel Polinomial } \\
\hline WM & Esquema & Error de Clasificación (\%) \\
bior37 & 2 & 3,57 \\
bior39 & 1 & 3,42 \\
rbio31 & 2 & 2,71 \\
\hline
\end{tabular}

WM: Wavelet madre. Fuente propia.

Tabla 6. Resultados de clasificación con la máquinas de soporte vectorial con kernel función de base radial.

\begin{tabular}{ccc}
\hline \multicolumn{3}{c}{ Resultados de clasificación MSV kernel Función de } \\
Base Radial \\
\hline WM & Esquema & Error de Clasificación (\%) \\
bior22 & 2 & 3,71 \\
bior33 & 2 & 3,85 \\
rbio31 & 2 & 3,00 \\
\hline
\end{tabular}

WM: Wavelet madre. Fuente propia 


\section{CONCLUSIONES}

Resulta significativo evaluar la capacidad de caracterización de las señales de interés con la DWT, independientemente si son latidos cardiacos o patrones de otro tipo, la transformada wavelet discreta tiene dos parámetros a definir, primero está la cantidad de niveles de descomposición que para el caso de la señales cardiacas, suelen ser hasta 4; respecto a las wavelet madre como vimos, hay 54 posibilidades (Incluyendo el orden), sin contemplar wavelets madre que se pueden crear a conveniencia; por lo que como se vio en la investigación aquí documentada, vale la pena hacer este tipo de estudios, precisamente por los buenos resultados de clasificación obtenidos, que evidencian la buena capacidad de caracterización de una wavelet madre muy poco utilizada e incluso conocida como es la biortogonal inversa. La wavelet madre biortogonal inversa 3.1 mostró los resultados más altos de energía en los coeficientes de detalles, con los 5 tipos de latidos cardiacos y tal vez por esta particularidad en los componentes de detalles es que el Esquema 2, que incluye los detalles, fue con el que se lograron la mayoría de resultados satisfactorios con las dos máquinas de aprendizaje, de las cuales, la máquina de soporte vectorial con kernel polinomial mostró una mejor capacidad de identificación, ligeramente superior a la máquina de soporte vectorial con función de base radial.

\section{BIBLIOGRAFIA}

1. D. Gao, M. Madden, M. Schukat, D. Chambers and G. Lyons, "Arrhytmia identification from ECG signals with a neural network classifier based on a Bayesian framework". Department of information Tecnology. National university of Ireland, 2003.

2. M. Benitez, "Desarrollo e implementación de un algoritmo para la caracterización de los límites de forma de onda en un ECG usando wavelets". Escuela politécnica nacional. JIEE, 2005; 19.

3. G. Selvakumar, , K. Bhoopathy, B. Vhidambararajan, "Wavelet decomposition for detection and classification of critical ECG arrhythmias", 8th WSEAS Int. Conference on Mathematics and Computers in Biology and Chemistry, 80 - 84, Vancouver, Canada, 2007.

4. G. Castellanos, J. Godino, E. Delgado, "Análisis acústico sobre señales de auscultación digital para la detección de soplos cardíacos", Avances en sistemas e informática, 2007; 4(3), 171 - 182.

5. A. Orozco, P. Muñoz, "Identificación de latidos cardiacos y desconocidos utilizando esquemas de clasificación en cascada", Scientia Et Technica, 2011; 48, 173 - 178.

6. Y. Wu, R. Rangayyan, Y. Zhou, S. Ng," Filtering electrocardiographic signals using an unbiased and normalized adaptive noise reduction system", Med Eng Phys 2009; 31(1): 17-26.

7. M. Vetterli, "Wavelet and filter banks: theory and designs". IEEE transactions on signal processing, 2002.

8. G. Garcia, R. Velandia, E Barón, "Algoritmo de reducción de ruido en señales de electroencefalografía utilizando la DWT”, Umbral científico junio número 008. Fundación Universitaria Manuela Beltrán, 2006.

9. C. Rengifo, G. Castellanos, R. Henao, "Aprendizaje activo en la identificación de cardiopatía isquémica", Grupo control y procesamiento de digital de señales. Universidad nacional de Colombia, Manizales, 2006.

10. A.Ordoñez, C. Castejón, J García, "Selección del nivel de descomposición WPT mediante el análisis de la sensibilidad de la energía relativa Wavelet", Dpto. Ing. Mecánica. Grupo MaqLab. Universidad Carlos III de Madrid, 2010.

11. I. Aldana, "Detección De Fallos En Rodamientos Mediante Análisis De Energía Y Lógica Difusa", Universidad Carlos III de Madrid, España, Departamento de Ingeniería mecánica, 2009.

12. Goldberger, A., y otros 9 autores, (2000); Components of a New Research Resource for Complex Physiologic Signals, Circulation electronic pages, 101(23), 215 - 220, Boston, USA.

13. Kuzilek, J., Spilka, J., Kremen, V., Lhotska, L., An Automatic Method for Holter ECG Denoising Using ICA, Proceedings of 4th International Symposium on Applied Sciences in Biomedical and Communication Technologies, 1 - 5, New York, USA, 2011. 
14. V. Montes, C. Guarín, G. Castellanos, "Extracción de características de ECG basadas en transformaciones no lineales y wavelets", Ingeniería e investigación, 2005; 25 (3), 39 - 48.

15. V. Kecman, "Support vector machines basics", School of Engineering. University of Auckland, Report 616, 1 - 58, Auckland, Nueva Zelanda, 2004.

16. R. Rengifo, L. Jiménez, "Máquinas De Soporte Vectorial”. Fundación Universitaria Konrad Lorenz. Facultad De Matemáticas E Ingenierías. Ingeniería De Sistemas. Bogotá, Colombia, 2005. 